\title{
Using Ultrasonic Reflection Resonance to Probe Stress Wave Velocity in Assemblies of Spherical Particles
}

\author{
Min Yu, Member, IEEE, Tom Reddyhoff, Daniele Dini, Andrew Holmes, Member, IEEE, and \\ Catherine O'Sullivan
}

\begin{abstract}
A high-sensitivity method to measure acoustic wave speed in soils by analyzing the reflected ultrasonic signal from a resonating layered interface is proposed here. Specifically, an ultrasonic transducer which can be used to both transmit and receive signals is installed on a low-high acousticimpedance layered structure of hard PVC and steel, which in turn is placed in contact with the soil deposit of interest. The acoustic impedance of the soil (the product of density and wave velocity) is deduced from analysis of the waves reflected back to the transducer. A system configuration design is enabled by developing an analytical model that correlates the objective wave speed with the measurable reflection coefficient spectrum. The physical viability of this testing approach is demonstrated by means of a one-dimensional compression device that probes the stress-dependence of compression wave velocity of different sizes of glass ballotini particles. Provided the ratio of the wavelength of the generated wave to the soil particle size is sufficiently large the data generated are in agreement with data obtained using conventional time-of-flight measurements. In principle, this high-sensitivity approach avoids the need for the wave to travel a long distance between multiple transmitterreceiver sensors as is typically the case in geophysical testing of soil. Therefore it is particularly suited to in-situ observation of soil properties in a highly compact setup, where only a single transducer is required. Furthermore, high spatial resolution of local measurements can be achieved, and the data are unaffected by wave attenuation as transmitted in soil.
\end{abstract}

Index Terms - ultrasonic reflection, geotechnical test, granular media, wave speed, soil stress.

\section{Introduction}

$\mathrm{T}$ HE small strain or elastic stiffness of soil is important in geotechnical engineering to predict ground deformations during and after construction and dynamic behaviour associated, for example, with site response to earthquakes. In practice, the compression wave (P-wave) and shear wave (Swave) velocities are often measured and used to obtain the

Manuscript received [Month] [Day], [Year]. This work was supported by the Natural Environment Research Council (NERC) under Grant NE/T010983/1

Min Yu (corresponding author, email: m.yu14@imperial.ac.uk), Tom Reddyhoff (email: t.reddyhoff@imperial.ac.uk) and Daniele Dini (email: d.dini@imperial.ac.uk) are with the Department of Mechanical Engineering, Imperial College London, SW7 2AZ London.

Andrew Holmes (email: a.holmes@imperial.ac.uk) is with the Department of Electrical and Electronic Engineering, Imperial College London, SW7 2AZ London.

Catherine O'Sullivan (email: cath.osullivan@imperial.ac.uk) is with the Department of Civil and Environmental Engineering, Imperial College London, SW7 2AZ London. constrained and shear moduli of soil specimens, according to elastic theory [1]. Geophysical methods based on stress wave transmission which measure the time taken for a wave to travel between a transmitter and a receiver have been widely employed in laboratory experiments [2]-[7] and in-situ testing [8]-[9] to determine the elastic wave velocities. In the laboratory, a pair of piezoceramic bender (or extender) elements, comprising a source element and a receiver element, are configured at opposite ends or sides of a sample to trace shear or compression wave transmission in shear mode. The established field seismic testing methods all involve use of separate sources and receivers. However, the need to use both a receiver and transmitter limits the nature of the data acquired; the measurements will reflect the ensemble properties along the path traversed by the wave and data reflecting local soil properties is not provided; this limitation is particularly relevant to in-situ soil wave measurements. Wave attenuation and dispersion make the received signals sensitive to noise so that in some cases no usable data are captured, especially in thicker 
soil samples and in the case of high frequency wave propagation. In laboratory tests, wave reflection from the side boundaries of a soil specimen container [10]-[11], can interfere with wave signal analysis (this usually occurs in the case of low-frequency signals and low width to height ratios).

A number of in-situ geophysical testing approaches have been proposed. For near-surface soils, a wave reflection technique captures the reflected wave at an interface of two different media using a circular ultrasonic waveguide to measure the speed of $\mathrm{P}$-waves and $\mathrm{S}$-waves, which propagate and reflect along a bar embedded into the soil [12]. During tunnelling, three-component geophones can be used to record seismic signals from the reflectors ahead of the tunnel face, creating a three-dimensional high-resolution seismic image [13]. Off-shore, oblique incident P-wave signals can be pulsed from ships into the sea, so that both reflected P-wave and the converted S-wave are detected by receivers (each consisting of one hydrophone and three orthogonally oriented geophones) located on the seabed in order to establish the stratigraphy underneath [14]. Ultrasound reflection has also been used to monitor soil saturation and soil erosion [15]-[16]. Other representative non-destructive testing techniques, including electrical permittivity (to determine the soil moisture [17]-[18]) and acoustics emission (to detect particle breakage and so on [19]), have been recently employed to estimate the geophysical properties. In soil mechanics element testing, ultrasonic resonance may be an alternative solution to the time-of-flight method - stress wave is pulsed to excite a soil deposit, the responded resonant frequencies of which are recorded to estimate soil properties such as elastic moduli [20].

Moreover, frequency domain analysis of the ultrasonic reflection has also been widely employed in other areas including medical devices (e.g., medical imaging, thermal therapy [21]) and tribological systems (e.g., probing the variation in thickness and viscosity of the lubricant film between two metal surfaces [22]-[27]). In these applications, a resonating or matching layer is usually introduced to achieve desirable reflection wave spectrum and improve measurement quality [21], [25]. It remains to be demonstrated that this approach can be applied to granular materials, acknowledging the issues: i) the elastic wave velocity depends on the stress level and material state, ii) dispersion occurs and iii) granular materials act as a filter to high frequency ultrasonic waves.

This paper proposes a novel method that induces highfrequency ultrasonic reflection resonance that can measure acoustic impedance and hence probe the elastic velocities in granular media. The key advantages of the method are: i) there is only one sensor with a highly compact configuration (when a high-frequency ultrasonic transducer is used); ii) the data are highly sensitive to any variation of wave speed due to the characteristics of wave reflection resonance, iii) the measurement is independent of the wave attenuation and dispersion effects; and iv) boundary effects on wave propagation are avoided. This technique is deemed to be suitable for both laboratory and in-situ wave speed measurements, for example, it could be included in a cone penetration test (CPT) device. The practical viability is validated through a one-dimensional compression soil cell, while the method robustness is further assessed with ranged ratios of wavelength-to-particle size. The main contributions of this study are:

- conceptualization of ultrasonic reflection resonance for a high-resolution impedance measure in granular media,

- development of an analytical model to characterize the resonance behavior and the sensitivity to material parameters

- experimental validation with the proposed method compared to the conventional time-of-flight method,

- robustness assessment of the proposed method in terms of applicable particle sizes.

\section{ThEORETICAL BASIS ANd ANALyticAl MODEL}

This section outlines the theory upon which the ultrasonic reflection measurement of soil acoustic impedance is based.

\section{A. Wave Transmission and Reflection Background}

Consider a system where there are two layers (layer 1 and layer 2) which have different acoustic impedances, $z_{1}$ and $z_{2}$ respectively. Referring to Fig. 1-a, when an incident stress wave encounters the interface between layer 1 and layer 2 (which is normal to the wave propagation direction), it will be partially reflected and partially transmitted. The reflection coefficient, $R$, is determined by the acoustic impedance discrepancy of the two layers [1] and given as:

$R=\frac{z_{2}-z_{1}}{z_{2}+z_{1}}$

where $z_{1}=\rho_{1} c_{1}$ and $z_{2}=\rho_{2} c_{2}$ are the acoustic impedances, $\rho_{1}$ and $\rho_{2}$ are the material densities and $c_{1}$ and $c_{2}$ are the wave propagation velocities in layers 1 and 2 respectively.

If an incident wave is transmitted into a three-layered structure (shown in Fig. 1-b), the overall reflection coefficient will be a complex number with both the amplitude and the phase angle determined by the materials' acoustic properties and the middle layer (layer 2) thickness, mathematically given as [28]:

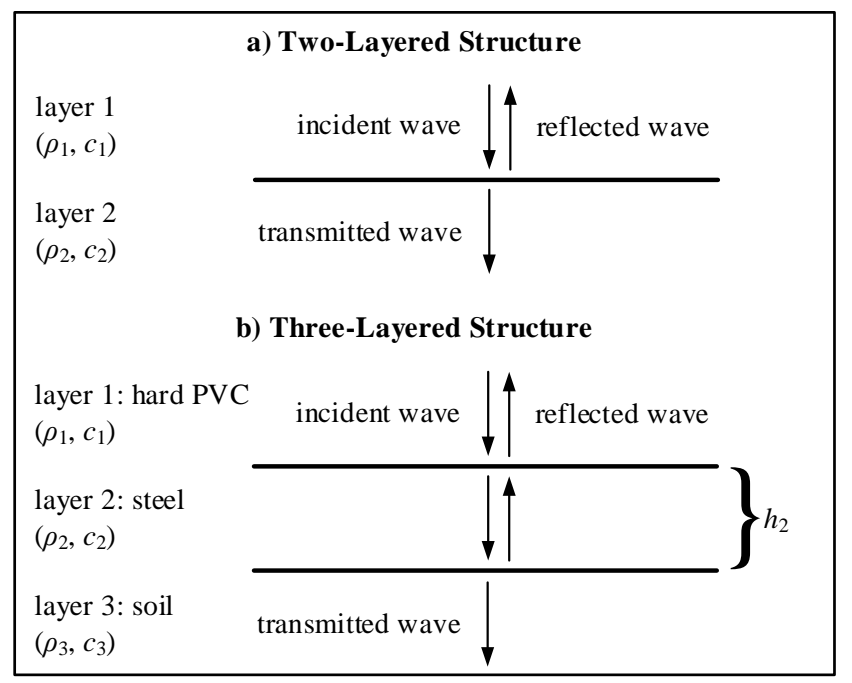

Fig. 1. Phenomenon of wave transmission and reflection into a) twolayered structure and b) a three-layered structure, where layers 1,2 and 3 correspond to hard PVC, steel and soil in the present work. 
$R=\frac{\left(z_{1}+z_{2}\right) \cdot\left(z_{2}-z_{3}\right) \cdot e^{\left(-i 2 \pi f h_{2} / c_{2}\right)}-\left(z_{2}-z_{1}\right) \cdot\left(z_{2}+z_{3}\right) \cdot e^{\left(-i 2 \pi f h_{2} / c_{2}\right)}}{\left(z_{2}-z_{1}\right) \cdot\left(z_{3}-z_{2}\right) \cdot e^{\left(-i 2 \pi f h_{2} / c_{2}\right)}+\left(z_{1}+z_{2}\right) \cdot\left(z_{2}+z_{3}\right) \cdot e^{\left(-i 2 \pi f h_{2} / c_{2}\right)}}$,

where $f$ is the wave frequency, $z_{1}, z_{2}$ and $z_{3}$ are the acoustic impedance of layers 1, 2 and 3 respectively, and $h_{2}$ and $c_{2}$ are the thickness and the wave speed of layer 2 .

\section{B. Analytical Modelling and Sensitivity Analysis}

The above three-layered expression for the reflection coefficient can be utilized to measure soil wave speed, provided the soil density is known. Referring to Fig. 1-b, both the materials comprising layers 1 and 2 and the thicknesses of these layers should be selected in order to maximize the reflection coefficient sensitivity to variations in the wave speed, while other practical factors (e.g. the material strength and durability) are taken into account to ensure implementation viability. Fig. 2-a shows the variation of the reflection coefficient amplitude $|R|$ with respect to the product of the wave frequency $(f)$ and the layer 2 thickness $\left(h_{2}\right)$, for a three-layered structure comprising hard PVC as layer 1, steel as layer 2 and a layer 3 made of soil. It can be seen that the minimum values of $|R|$, which are associated with resonance, are very sensitive to variations in $c_{3}$ (assuming a constant density). The resonance of the reflected signal occurs when half of the wavelength in layer $2\left(\lambda_{2}\right)$ equals to the layer 2 thickness $\left(h_{2}\right)$, that is:

$f_{r} \cdot h_{2}=N \cdot \frac{c_{2}}{2}$

where $f_{r}$ is the denoted as the resonant frequency, and $N=1,2$, ... By combining (2)-(3), the minimum amplitude of the
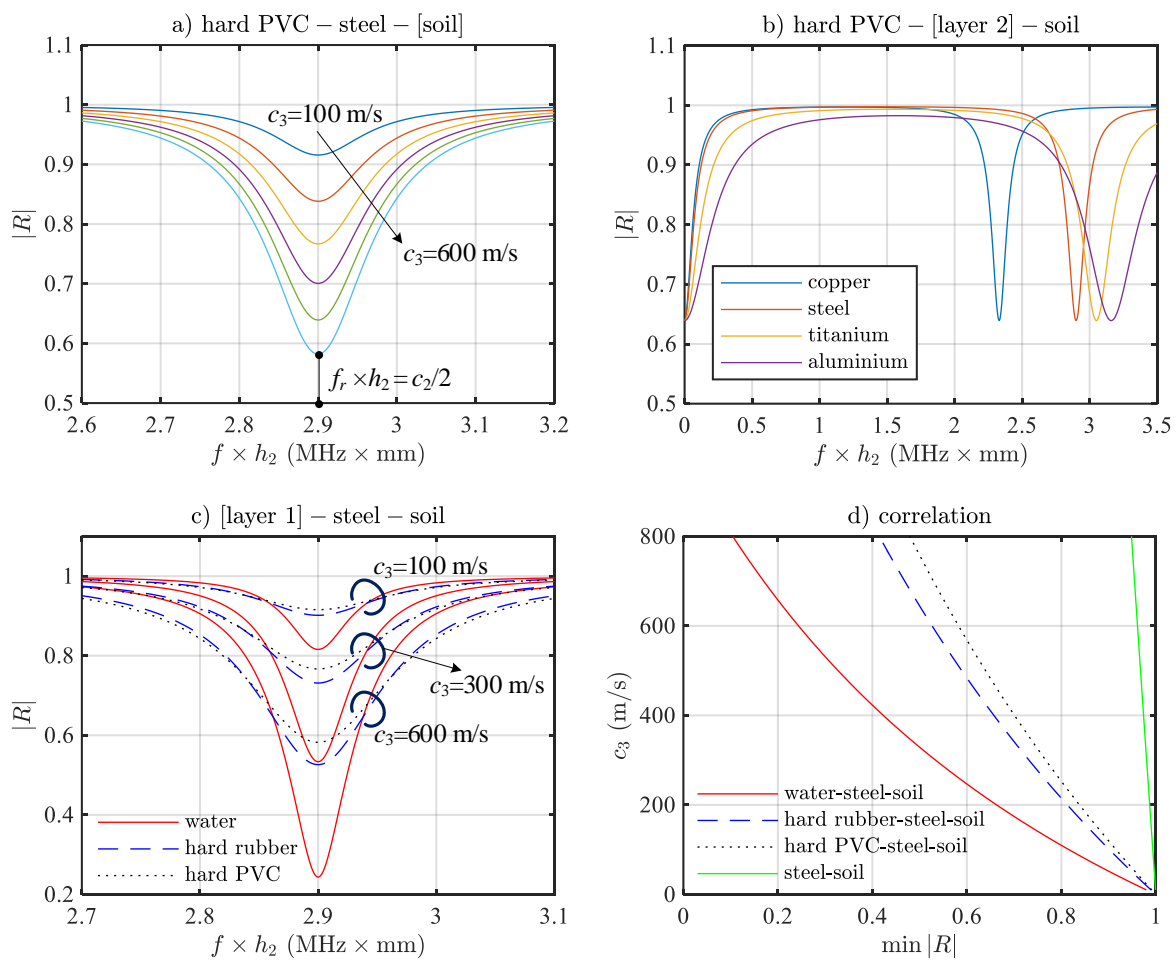

reflection coefficient, which occurs at the resonant frequency $\left(f_{r}\right)$, can be obtained:

$\min |R|=\frac{z_{1}-z_{3}}{z_{1}+z_{3}}$.

Equation (4) indicates that $\min |R|$ is independent of layer 2 and depends only upon $z_{1}$ and $z_{3}$ (also shown in Fig. 2-b). Furthermore, the soil wave speed $\left(c_{3}\right)$ can be given as:

$c_{3}=\frac{\rho_{1} c_{1}}{\rho_{3}} \cdot \frac{1-\min |R|}{1+\min |R|}$,

In contrast to the geophysical methods that rely on stress wave transmission, the soil density is required to enable prediction of the wave velocity in layer 3. The material selection of the layers 2 and 3 are justified as follows: in practice layer 2 should be a metal material that is strong while its thickness needs to be properly designed to satisfy the resonance condition in (3). Moreover, layer 1 should be made of a low-acoustic-impedance material, otherwise, the ultrasonic reflection will be insensitive to the objective variable of the soil wave speed $\left(c_{3}\right)$ - this is similar to the performance of a twolayered structure of "steel-soil" (shown as the green line in Fig. 2-d). According to Fig. 2-c and -d, the selection of either water or hard rubber for layer $1 \mathrm{can}$, in principle, enable better measurement resolution compared to hard PVC. However, water can only transmit $\mathrm{P}$-waves and not $\mathrm{S}$-waves and requires non-trivial physical packaging and assembly. In contrast, hard rubber suffers from poor machinability, and is incompatible with the requirement for a highly smooth surface with low-level

Fig. 2. Analytical model of the reflection coefficient amplitude $|R|$ and sensitivity analysis: a) a three-layered structure of "hard PVC-steel-[layer 3]", where the layer 3 is a soil sample with the P-wave speed assumed $c_{3} \in[100,600] \mathrm{m} / \mathrm{s}$; b) a three-layered structure of "hard PVC-[layer 2]-soil", where typical metal materials of aluminium, copper, steel, titanium are selected respectively as the middle layer 2 and the soil P-wave speed is fixed at $c_{3}=500 \mathrm{~m} / \mathrm{s} ; \mathrm{c}$ ) a three-layered structure of "[layer 1]-steel-soil", where the layer 1 is with low-acoustic-impedance material of water, hard rubber and hard PVC respectively while $\left.c_{3}=[100,300,600] \mathrm{m} / \mathrm{s} ; \mathrm{d}\right)$ correlation between the measurable variable of min $|R|$ and the objective of soil P-wave speed $c_{3}$. 
roughness necessary for the interface between layer 1 and 2 in order to guarantee sufficient wave propagation intensity. Therefore, hard PVC is finally selected as the material for layer 1 , the other side of which will be attached by an ultrasonic sensor for signal pulsing and receiving. Other similar lowacoustic-impedance materials, for example, Perspex, are alternatives for the layer 1, showing similar performance.

Furthermore, data on Fig. 2-d clarify that a three-layered "hard PVC-steel-soil" system, instead of a simple "steel-soil" configuration, is needed to obtain sufficient measurement resolution. If the soil wave speed is assumed to vary from $0 \mathrm{~m} / \mathrm{s}$ to $800 \mathrm{~m} / \mathrm{s}$, the corresponding reflection coefficient with "steelsoil" ( $R$ is a constant value in this case, thus $\min |R|=R$ ) exhibits only a small change (from 1 to 0.95 ); in contrast, the proposed resonance technique using the "hard PVC-steel-soil" significantly improves the measurement resolution, with the

\section{TABLE I}

ACOUSTIC PROPERTIES OF DIFFERENT MATERIALS IN THE ANALYTICAL MODEL

\begin{tabular}{llll}
\hline \hline & $\begin{array}{l}\text { Density } \\
\left(\mathrm{kg} / \mathrm{m}^{3}\right)\end{array}$ & $\begin{array}{l}\text { P-Wave Speed } \\
(\mathrm{m} / \mathrm{s})\end{array}$ & $\begin{array}{l}\text { Acoustic Impedance } \\
\left(10^{6} \mathrm{~kg} \cdot \mathrm{m}^{-2} \cdot \mathrm{s}^{-1}\right)\end{array}$ \\
\hline Hard PVC & 1450 & 2350 & 3.41 \\
Water & 1000 & 1480 & 1.48 \\
Steel & 7810 & 5800 & 45.3 \\
Copper & 8960 & 4660 & 41.6 \\
Aluminium & 2700 & 6320 & 17.1 \\
Titanium & 4540 & 6100 & 27.7 \\
Soil & 1500 & $100-600^{*}$ & $0.15-0.90$ \\
\hline
\end{tabular}

* A wide range of soil wave speed is assumed to demonstrate capability of the ultrasonic resonance method.

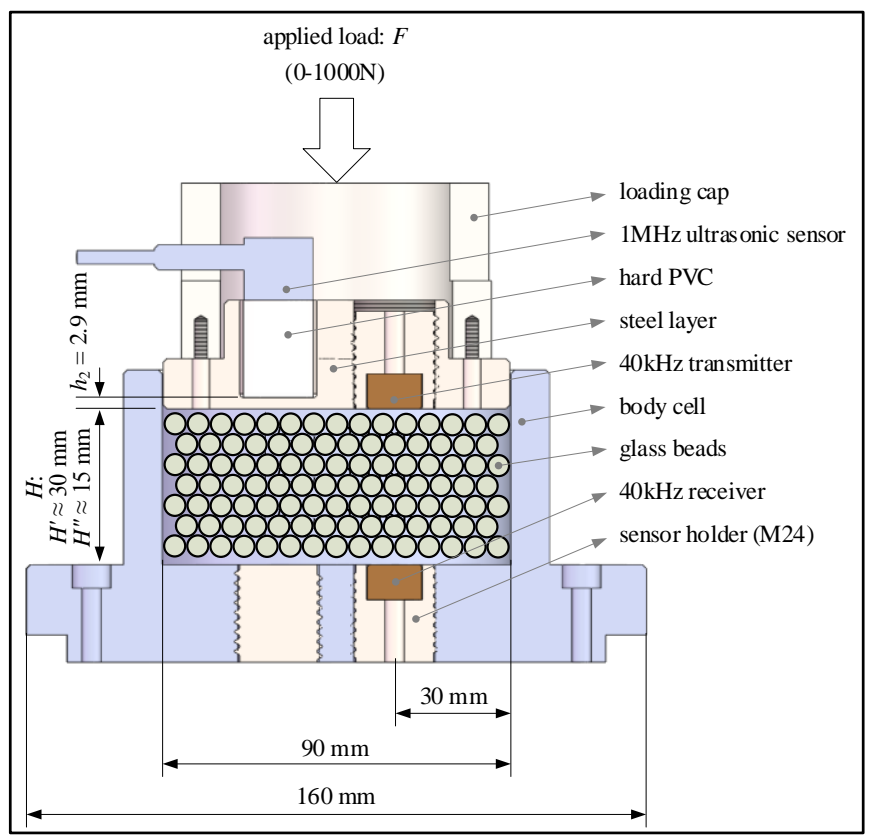

Fig. 3. Schematic of the test setup for wave speed measurements in granular media (glass ballotini), where both the proposed ultrasonic resonance set-up (with a $1 \mathrm{MHz}$ ultrasonic sensor) and the conventional time-of-flight set-up (with a $40 \mathrm{kHz}$ transmitter-receiver pair) are installed. $H$ is the thickness of the soil specimens (two different thickness, $H^{\prime} \approx 30 \mathrm{~mm}$ and $H^{\prime \prime} \approx 15 \mathrm{~mm}$, are tested separately to enable an incremental time-of-flight measurement, as detailed in Section III.B). measurable $\min |R|$ varying from 1 to 0.48 .

Based on the above, a three-layered structure of "hard PVCsteel-soil" is adopted in this study to probe the variation of the soil wave speed, in order to ensure practical viability in terms of optimum measurement resolution, structural strength. In addition, the acoustic properties of the associated materials are listed in Table I.

\section{EXPERIMENTAL SETUP AND RESULTS DISCUSSION}

\section{A. Experimental Design and Setup}

Oedometer testing has been widely utilized to investigate geotechnical properties of a consolidated soil sample by applying a varied load [2]-[3], [6]-[7]. We expect the stiffness, and hence the elastic wave velocity, of soil, or any granular material, to vary with pressure [29]-[30] and so to demonstrate the viability of this testing approach we sought first to establish whether the proposed method could capture the stress dependency of the elastic wave speed. As illustrated in Fig. 3, this study used a one-dimensional compression cell similar to an oedometer but with a smaller diameter-to-height ratio, having a diameter of $90 \mathrm{~mm}$ and a typical specimen height of $30 \mathrm{~mm}$. This cell could be placed in an existing Universal Mechanical Tester (UMT [31]). A vertical force of up to 1000 $\mathrm{N}$ could be applied to soil specimens to give vertical stresses of up to $157 \mathrm{kPa}$. The vertical displacement the top of the soil samples was measured with an in-built LVDT sensor. A highfrequency ultrasonic transducer ( $1 \mathrm{MHz}$, as supplied by [32]) is employed to pulse an incident $\mathrm{P}$-wave signal and also to receive the reflected signal (delayed with an interval of the forward and backward travelling time); the thickness of the middle steel layer is $2.9 \mathrm{~mm}$ according to the resonance criteria in (3) (i.e., this thickness ensures the produced resonant frequency, $f_{r}$, falls close to the centre frequency of the transducer $f_{c}$ ); a hard PVC cylinder is coupled to establish the three-layered structure of "hard PVC-steel-soil", where coupling gel is applied onto both sides of the hard PVC to improve the signal intensity of wave propagation. The thickness of the hard PVC (minimum $25 \mathrm{~mm}$ is required in the present application) is carefully selected to eliminate any overlap or interference between the reflected wave and its adjacent signals, as detailed later in Fig. 6-b in Section III.C. The selection of the high-frequency $1 \mathrm{MHz}$ transducer leads to a highly compact sensing package, which is well suited for inclusion in in-situ testing devices such as a CPT sleeve. In contrast, lower frequency ultrasonic transducers would require relatively thicker steel layer and use of hard PVC assembly. To enable verification of the data acquired using the wave reflection process, stress wave transmission through the sample was also measured using a $40 \mathrm{kHz}$ transmitter and receiver pair. The threaded M24 sensor holders were designed to increase the flexibility of the overall apparatus configuration, enabling easy sensor replacement. Glass ballotini, different sizes of which correspond to different categories of soils/sands, are often used as model soils [33]-[34]. Therefore ranged sizes of glass ballotini, from the minimum diameter of $0-50 \mu \mathrm{m}$ to the maximum diameter of 1.7-2.1 mm, are tested separately, which are believed enough in this proof to study the robustness of the concept.

The experimental procedures used to acquire the wave transmission data and the reflection resonance data are shown 
in the flowchart in Fig. 4, and detailed in Sections III.B and III.C below.

\section{B. Wave Speed Measurements Using Transmitted Signal Data}

Fig. 5-a plots the transmitted and received signals for the transmission test sensors used for validation. The wave propagation speed cannot be accurately obtained by simply dividing the soil thickness $(H)$ with the peak time difference (between the transmitter and the receiver waveforms). This is because: i) there is a travel time associated with the passage of the waves within sensors themselves (time-of-flight tests with a varied thickness of water samples - where the water wave speed is known - are also performed, the results are provided in Fig. A1 in the Appendix, showing a travel time of around $2.1 \times 10^{-5} \mathrm{~s}$ within sensors, which cannot be ignored in soil wave speed measurements as the soil specimen layer is relatively thin); and ii) the signals generated by the piezoelectric sensors are also slightly affected by the applied soil stress. To compensate for these errors and uncertainties, an incremental time-of-flight is used, where at least two sets of tests need to be performed (two soil specimens are thus separately prepared, with the prepared soil thickness $H$ being around $H^{\prime} \approx 30 \mathrm{~mm}$ and $H^{\prime \prime} \approx 15 \mathrm{~mm}$ respectively. The actual values of $H^{\prime}$ and $H^{\prime \prime}$ can be accurately measured by an in-built LVDT sensor during the loading tests). Fig. 5-b shows the variation of the peak time $t^{\prime}$ and $t^{\prime \prime}$ at the receiver ends with $H=H^{\prime}$ and $H=H^{\prime \prime}$ respectively. Eventually, the calibrated wave speed $(\bar{c})$ is obtained by dividing the soil thickness increment $\left(H^{\prime}-H^{\prime \prime}\right)$ by the receiver peak time increment $\left(t^{\prime}-t^{\prime \prime}\right)$, as plotted in Fig. 5-c. Data were collated for two samples with the actual value of $H^{\prime}=32.6 \mathrm{~mm}$ and $H^{\prime \prime}=13.1 \mathrm{~mm}$ where the load was varied from 50 to 1000 $\mathrm{N}$ to give a stress range of $8-157 \mathrm{kPa}$. The expected pressure dependency of the wave speed is clearly evident in Fig. 5-c.

\section{Wave Speed Measurements with Ultrasonic Resonance}

The proposed reflection resonance method follows the flowchart of the procedures in Fig. 4-b. The example results shown here are for a soil specimen of small glass ballotini (diameters in the range of $0-50 \mu \mathrm{m}$ ). To calculate the reflection coefficient $(R)$, a reference signal of "hard PVC-steel-air", is firstly required. These data enable calibration of $R=1$ with the zero-acoustic-impedance of air by cancelling out wave attenuation effect (in the hard PVC layer prior to acquiring any test signals for the "hard PVC-steel-soil" layer).

Fig. 6-a and -b show reflected wave recorded at the sensor for the two-layer "hard PVC-air" and the three-layer "hard PVC-steel-air" system in the time domain respectively, the amplitude of the latter is significantly reduced due to the intentionally produced resonance with "hard PVC-steel-air". The signal segment of $0-2.5 \times 10^{-4} \mathrm{~s}$ needs to be truncated, i.e. excluded from the frequency domain analysis, as this part of the signal is generated when the wave leaves the sensor and is immediately reflected from the interface between the ultrasonic sensor and the hard PVC. The signal that penetrates and reflects from the target structured interfaces ("hard PVC-steel-air") is recorded over the time segment of $2.5 \times 10^{-4}-5.5 \times 10^{-4} \mathrm{~s}$, as highlighted in red in Fig. 6-b and used later in the frequency domain analysis. The time required for forward and backward propagation is at least $2 h_{1} / c_{1}$, where $h_{1}$ is the thickness of the hard PVC and $c_{1}$ is its wave propagation speed, in this case this gives a value of $2.5 \times 10^{-4} \mathrm{~s}$ as $h_{1}=25 \mathrm{~mm}$. The thickness of the hard PVC layer must be carefully considered: if the thickness is insufficient, then the reflected wave will interfere with adjacent waves (which can be either the signal at $0-2.5 \times 10^{-4} \mathrm{~s}$ or a second echo from the PVC-steel interface commencing at around $5.5 \times 10^{-4} \mathrm{~s}$ ). On the other hand, if the hard PVC layer is too thick the reflected wave will be significantly attenuated, thus reducing the signal to noise ratio to an unacceptable level. The signal highlighted in red is extracted and a FFT is used to generate the data for the frequency domain response, as plotted in Fig. 6-c, where a resonance at $f_{r}=0.97 \mathrm{MHz}$ is clearly observed as compared to the non-resonant structure of "hard PVC-air". The small deviation from the nominal value of the resonant frequency, $1 \mathrm{MHz}$ as calculated using (3), is due to the parameter errors (in $c_{2}$ and $h_{2}$ ), however, this does not affect the measurements as long as the actual resonant frequency falls into the transducer frequency bandwidth. The test signal obtained using a "hard PVC-steel-soil" configuration with a varied load of 50-1000 N (equivalent to 8-157 $\mathrm{kPa}$ ), together with the reference signal from the "hard PVC-steel-air", in the

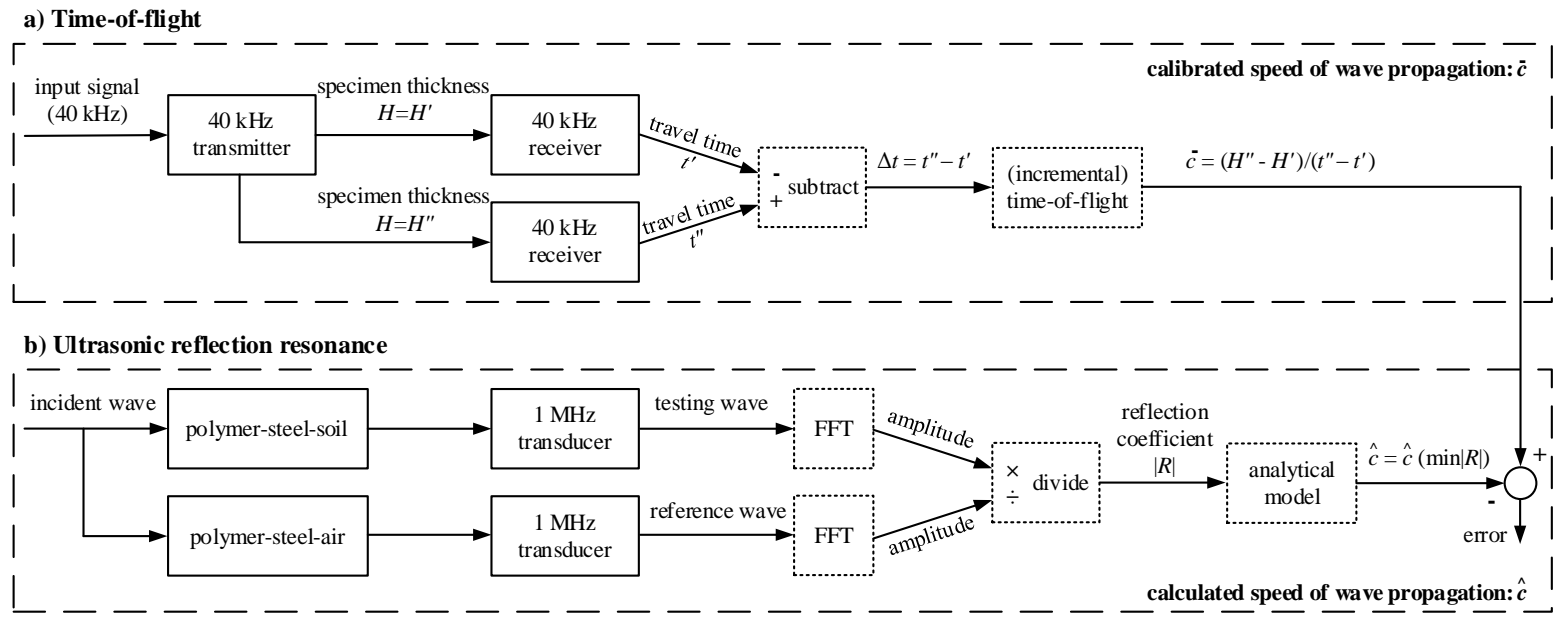

Fig. 4. Test procedures of implementing a) the conventional time-of-flight method (the upper dashed block) and b) the proposed ultrasonic resonance method (the lower dashed block) for the wave propagation speed measurements. 

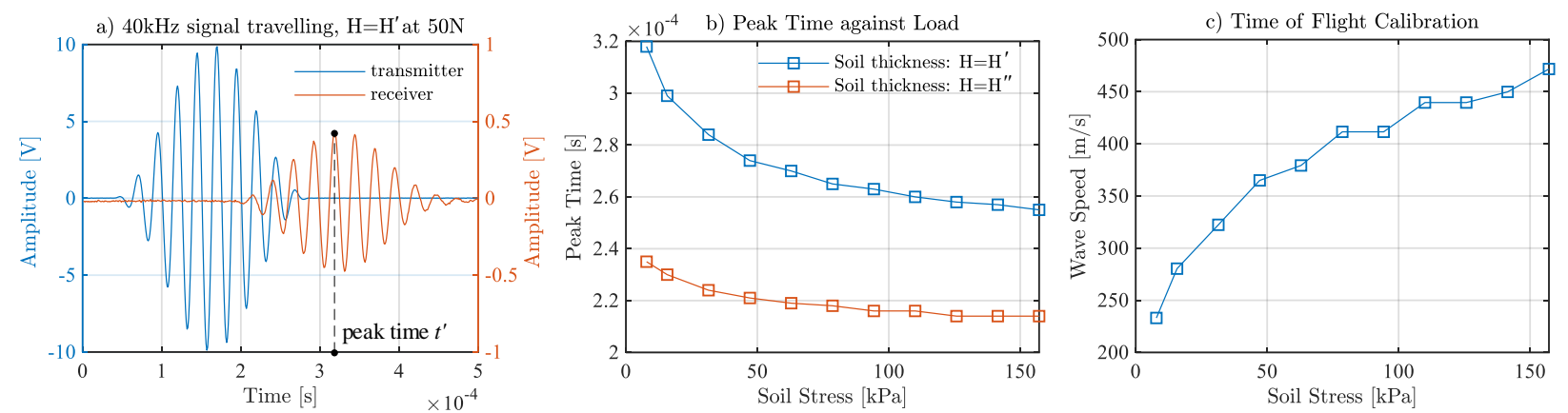

Fig. 5. Experimental results of wave speed measurements with the incremental time-of-flight method (for a soil deposit of $0-50 \mu m$ glass ballotini): a) an example of the $40 \mathrm{kHz}$ input transmitter signal (fixed throughout experiments) and the output receiver signal with the applied load $F=50 \mathrm{~N}$ (or $\sigma=8 \mathrm{kPa}$ ) and the soil specimen height $H=H^{\prime}$; b) the peak time $t^{\prime}$ and $t^{\prime \prime}$ (with the case of $H^{\prime}=32.6 \mathrm{~mm}$ and $H^{\prime \prime}=13.1 \mathrm{~mm}$ respectively, which are measured by a LVDT sensor) against the soil stress $(\sigma)$; and c) the time-of-flight calibrated wave speed $(\bar{c})$ against the soil stress $(\sigma)$, where $\bar{c}=\left(H^{\prime}-H^{\prime \prime}\right) /\left(t^{\prime}-t^{\prime \prime}\right)$.
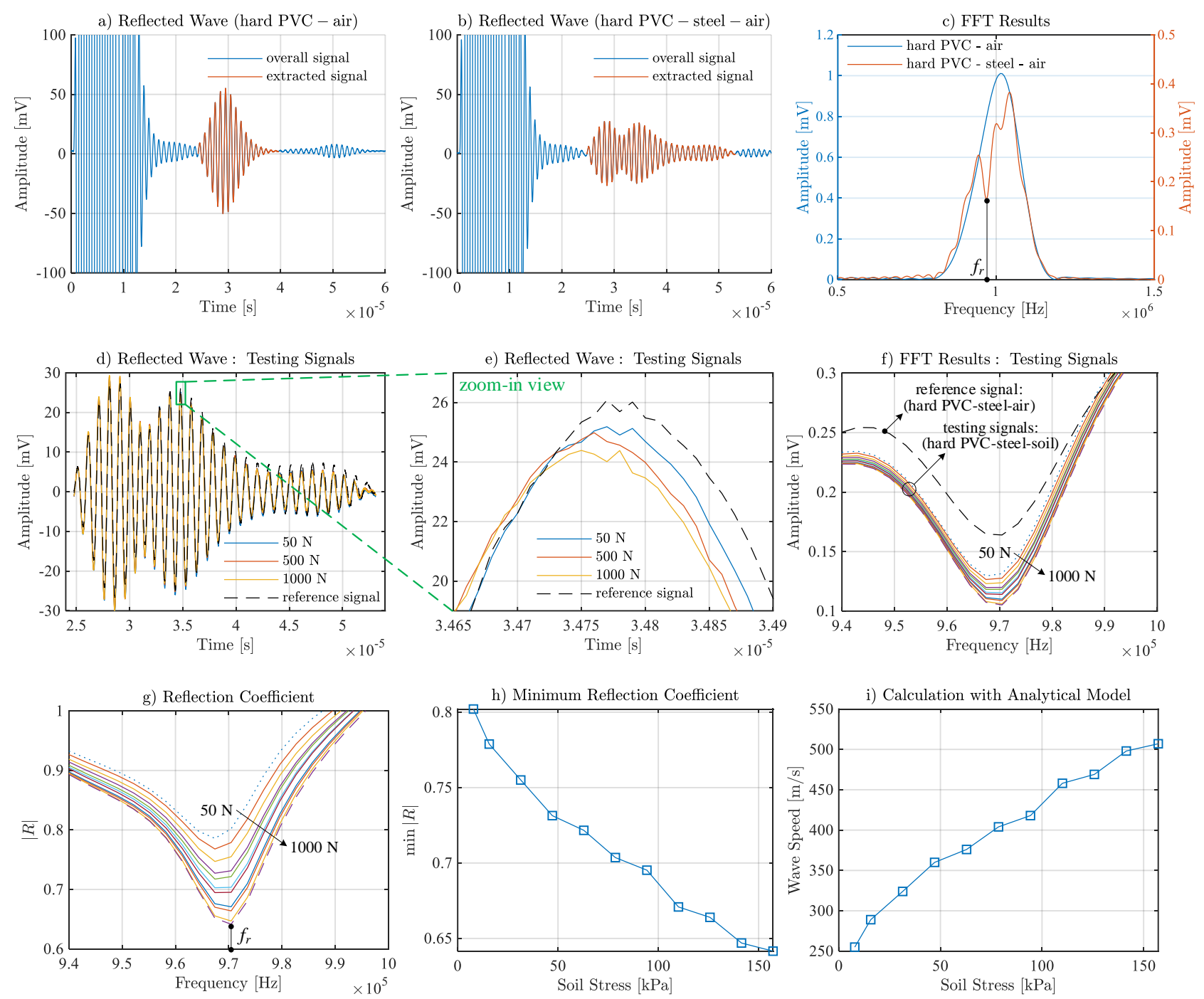

Fig. 6. Experimental results of wave speed measurements with the proposed reflection resonance method (for a soil deposit of $0-50 \mu m$ glass ballotini): a) the reflection wave with "hard PVC-air" in the time domain; b) the reference reflection wave with "hard PVC-steel-air" in the time domain; c) the amplitude of the reflection wave "hard PVC-air" and "hard PVC-steel-air" in the frequency domain. $f_{r}$ is the resonant frequency with a local minimum reflection wave amplitude, thus exhibiting a minimum value in the reflection coefficient amplitude spectrum (see theoretical analysis results with "hard PVC-steel-air" in Fig. 2-a, which also agrees with experiment results shown later in Fig. 6-g); d)-e) the testing signals with "hard PVCsteel-soil" in the time domain, where different soil loads are presented; f) the amplitude of the testing signals in frequency domain, where the soil load ranges $F \in[50,1000] \mathrm{N}$ or equivalent to $\sigma \in[8,157] \mathrm{kPa} ; \mathrm{g})$ the amplitude of the reflection coefficient in the frequency domain; $\mathrm{h}$ ) the minimum amplitudes of the refection coefficients $\left(\min |R|\right.$, which is at the resonant frequency $f_{r}$ ) with respect to the applied soil stress $(\sigma)$; and i) the calculated wave speed $(\hat{c})$ using the analytical model in (5). 

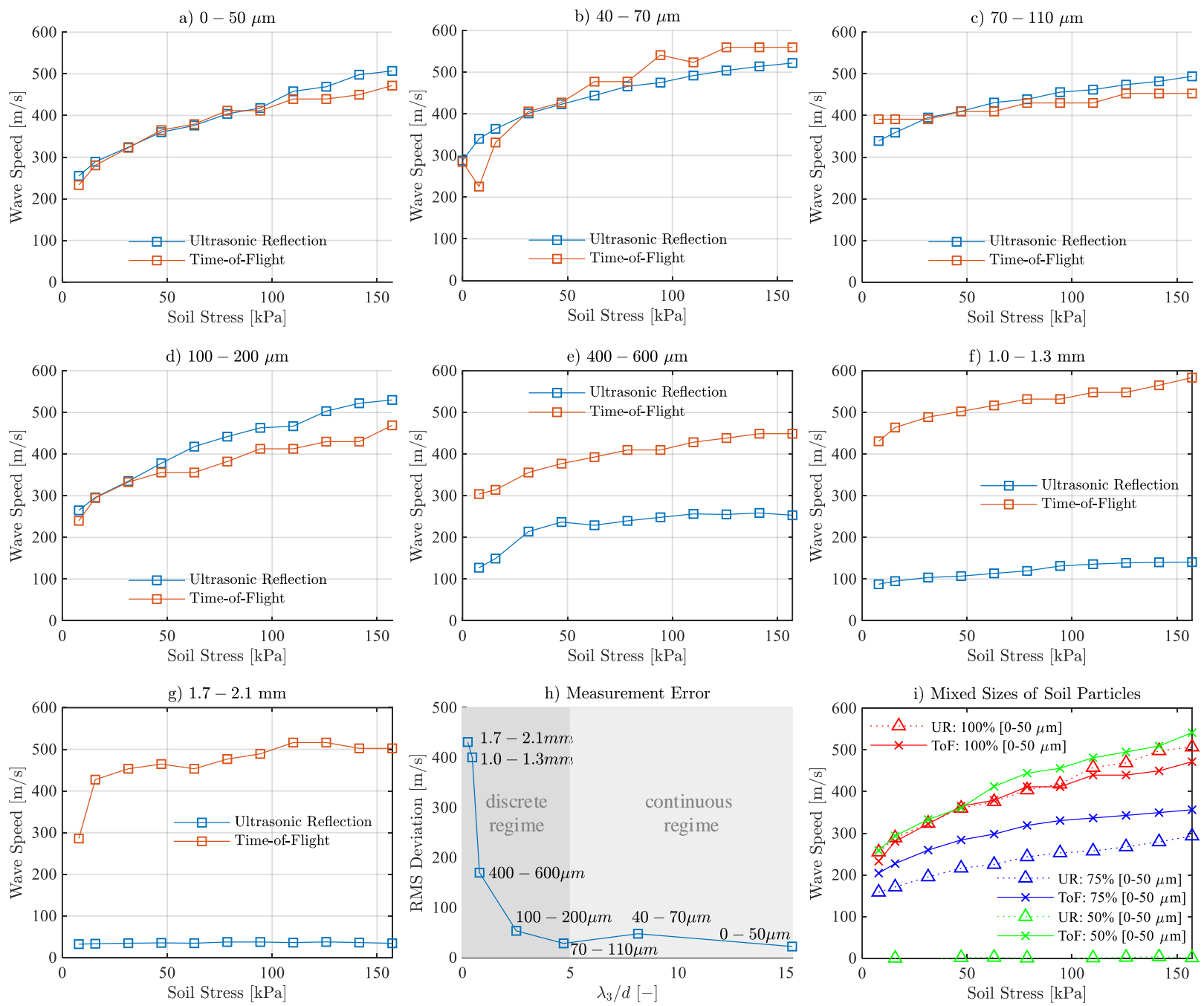

Fig. 7. Experimental results with different sizes of soil particles: a)-d) are with the small glass ballotini, where the wavelength is greater than the particle size; e)-g) are with large glass ballotini, where the wave wavelength is close to or less than the particle size; $h$ ) the RMS deviation (as compared to the time-of-flight measurement results) against $\lambda_{3} / d$ (where $\lambda_{3}$ is the wavelength in the soil layer while $d$ is the diameter of soil particles) to suggest the particle size influence; and i) a mixture of "continuous regime" glass ballotini $(0-50 \mu \mathrm{m})$ and "discrete regime" glass ballotini (1.7-2.1 $\mathrm{mm}$ ), where the percentage $100 \%, 75 \%$ and $50 \%$ refer to the proportion of $0-50$ "continuous regime" glass ballotini $0-50 \mu \mathrm{m}$, and the methods of ultrasonic reflection and time-of-flight are denoted by "UR" and "ToF" respectively.

time domain are presented in Fig. 6-d and -e, in the frequency domain are shown in Fig. 6-f. Amplitude spectrum signals are further used to calculate the amplitude of the ultrasonic reflection coefficient $(|R|)$, as plotted in Fig. 6-g, which is equivalent to the theoretical response shown in Fig. 2-a. The minimum amplitudes of these reflection coefficients, $\min |R|$, at the resonant frequency are then found and plotted with respect to the applied stress $(\sigma)$, as shown in Fig. 6-h. Finally, the objective variable of the soil wave speed $(\hat{c})$ is calculated using the analytical model developed in Section II.B, and shown in Fig. 6-i, where $\hat{c}$ continuously increases with respect to $\sigma$. It is worth noting that the soil density is assumed to be a constant as the vertical deformation of the glass ballotini during the loading tests is negligible, according to the LVDT measurements shown in Fig. A2 in the Appendix.

\section{Experimental Results and Discussion}

The time-of-flight calibrated soil wave speed $(\bar{c})$ with respect to the soil stress essentially agrees with the ultrasonic resonance measurements $(\hat{c})$, as shown in Fig. 7 -a to $-\mathrm{d}$, where different small diameters of glass ballotini (from 0-50 $\mu \mathrm{m}, 40-70 \mu \mathrm{m}, 70-$ $110 \mu \mathrm{m}$ to $100-200 \mu \mathrm{m})$ are tested separately to assess the robustness of the proposed method. In contrast, the ultrasonic resonance method begins to show significant measurement errors when dealing with larger particles (from 400-600 $\mu \mathrm{m}$, $1.0-1.3 \mathrm{~mm}$ to $1.7-2.1 \mathrm{~mm}$ ), as demonstrated by Fig. 7-e to -g. The particle size influence on the ultrasonic resonance measurement is further quantified in Fig. 7-h, which provides the RMS deviations (as compared to the time-of-flight measurement results) against $\lambda_{3} / d$ (where $\lambda_{3}$ is the wavelength in the soil layer while $d$ is the diameter of soil particles). It can be initially concluded that the proposed ultrasonic resonance method is applicable on the condition that $\lambda_{3}>5 d$, illustrated as the "continuous regime". In contrast, the proposed approach will be invalid once $\lambda_{3} / d$ falls within the "discrete regime". Additionally, experiment results with a mixture of "continuous regime" glass ballotini $(0-50 \mu \mathrm{m})$ and "discrete regime" glass ballotini $(1.7-2.1 \mathrm{~mm})$ are provided in Fig. $7-\mathrm{i}$, where it can be 
roughly seen that higher proportion of "discrete regime" glass ballotini may introduce larger measurement errors of the ultrasonic reflection method. The behavior of gap-graded granular materials (i.e., mixtures of coarse and cohesionless fine grains) is however highly complex, and depends upon i) the ratio of the large and small diameters and ii) volumetric proportion of the finer fraction [35], therefore further comprehensive investigation is required for the application of the ultrasonic reflection method in this scenario. The main parameters of the tested glass ballotini, as well as layer 1 of hard PVC and layer 2 of steel, are listed in Table II.

The above phenomenon of "discrete regime" is roughly illustrated and further explained in Fig. 8, which shows the wave propagation from a continuum media to a discrete media. The wavelength of a $1 \mathrm{MHz}$ signal in the steel layer and in the soil should be around $\lambda_{2}=5.8 \mathrm{~mm}$ and $\lambda_{3}=0.5 \mathrm{~mm}$, which is around $25 \%$ the diameter of the $2 \mathrm{~mm}$ glass ballotini itself. In this case, the wave from the interface with the soil side is a mixture of steel-air and steel-glass reflections and thus it is more likely to be randomly scattered [36]. This complexity

TABLE II

PROPERTIES OF THREE LAYERS IN EXPERIMENTAL TESTS

\begin{tabular}{llll}
\hline \hline & $\begin{array}{l}\text { Bulk Density } \\
\left(\mathrm{kg} / \mathrm{m}^{3}\right)\end{array}$ & $\begin{array}{l}\text { P-Wave Speed } \\
(\mathrm{m} / \mathrm{s})\end{array}$ & $\begin{array}{l}\text { Void Ratio } \\
(-)\end{array}$ \\
\hline Hard PVC & 1450 & 2350 & - \\
Steel & 7810 & 5800 & - \\
$\begin{array}{l}\text { Glass ballotini } \\
(0-50 \mu \mathrm{m})\end{array}$ & $1457^{*}$ & $250-550$ & $0.72^{* *}$ \\
$\begin{array}{l}\text { Glass ballotini } \\
(40-70 \mu \mathrm{m})\end{array}$ & 1450 & $250-550$ & 0.72 \\
$\begin{array}{l}\text { Glass ballotini } \\
(70-110 \mu \mathrm{m})\end{array}$ & 1490 & $250-550$ & 0.68 \\
$\begin{array}{l}\text { Glass ballotini } \\
(100-200 \mu \mathrm{m})\end{array}$ & 1515 & $250-550$ & 0.65 \\
$\begin{array}{l}\text { Glass ballotini } \\
(400-600 \mu \mathrm{m})\end{array}$ & 1530 & $250-550$ & 0.63 \\
$\begin{array}{l}\text { Glass ballotini } \\
(1.0-1.3 \mathrm{~mm})\end{array}$ & 1432 & $250-550$ & 0.75 \\
$\begin{array}{l}\text { Glass ballotini } \\
(1.7-2.1 \mu \mathrm{m})\end{array}$ & 1472 & $250-550$ & 0.70 \\
\hline
\end{tabular}

* Density of glass ballotini specimens are calculated as weight divided by the volume (with the soil thickness $H$ measured by a LVDT sensor).

** Void ratios of glass ballotini specimens are estimated as: (specific gravity - bulk density)/(bulk density), where the nominal value of specific gravity of glass ballotini is 2.5 . Moreover, the void ratios are considered to be constant in the present study, as the soil deformation during the loading tests is negligible (see in Fig. A2 in the Appendix).

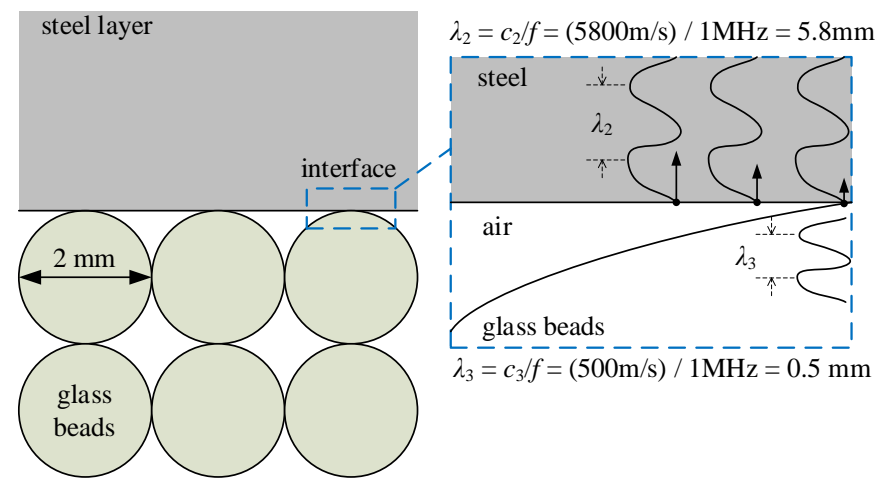

Fig. 8. Illustration of the wave reflection at the interface between the continuum steel layer and large soil particles $(2 \mathrm{~mm}$ diameter of glass ballotini). $\lambda_{2}$ and $\lambda_{3}$ are the wavelength in the steel and soil layers. makes (1), on which the analytical model is based, invalid. Numerical simulations are therefore required to further investigate the characteristics of wave transmission and reflection that is associated to discrete media. A similar phenomenon can be also found in the application of ultrasonic reflection when studying contact between rough surfaces, where the presence of large gaps at the interface causes the assumption that the wavelength is considerably greater than the gap size to break down [37]. On the other hand, the time-offlight measurements with $1.7-2.0 \mathrm{~mm}$ glass ballotini begin to suffer from noisy signals, as the $40 \mathrm{kHz}$ wave pulsed by the transmitter is significantly dispersed and attenuated before reaching the receiver, making the measurement results less reliable. Overall, to deal with large soil particles, both the timeof-flight and the reflection resonance methods need to adopt lower frequency sensors. The ratio of the wavelength to particle size seems to be the dominant factor for the applicability of the ultrasonic reflection method, while the reliability of the timeof-flight method can be additionally affected by soil thickness, soil stress and so on, as indicated in [1], [35]. Future work will investigate the robustness of the reflection resonance method through in-situ practice.

\section{CONCLUSION}

A novel method that produces high-frequency ultrasonic reflection resonance has been proposed to probe acoustic impedance and hence wave speed propagated in granular media. Specifically, a three-layered "hard PVC-steel-soil" configuration is established to maximise the sensitivity of the reflection to the variation of the wave speed, according to the analytical modelling and sensitivity analysis. Other practical factors, for example a high-strength metal layer (the steel material used in the present work) is required for in-situ testing to separate high-stress soils and packaged sensors, are also taken into account to ensure the implementation viability. To validate this concept, an experimental setup of one-dimensional compression is designed and developed to enable the measurements of the soil stress dependence of the longitudinal wave (P-wave) speed. Test results with different diameters of glass ballotini suggest the wave speed measurements using the proposed ultrasonic reflection method essentially agree with these from the conventional time-of-flight method. Despite the limitations that i) the soil density must be known and ii) the ratio of particle size to wavelength must be considered when selecting sensors, the proposed reflection resonance method has some advantages in the context of soil wave measurements:

- A single-end configuration with a highly compact system package, whereas the employment of a lower frequency ultrasonic transducer leads to a thicker steel layer-hard PVC assembly.

- High sensitivity to the variation of wave speed, due to a low-acoustic-impedance layer (i.e. the hard PVC) coupled with the thickness of a steel layer properly selected.

- The attenuation associated with wave propagation in the soil, which normally makes the conventional time-of-flight measurements sensitive to signal noise, does not affect the capture of the reflection signal.

- High spatial resolution of local measurements can be achieved. 
- As a high-frequency ultrasonic wave decays rapidly when penetrating soils even by a short distance, it does not suffer the "boundary effect" (the wave propagation striking at the boundary of a soil body cell and hence contaminating the signal at the receiver end in the time-of-flight configuration).

All of the above advantages suggest the proposed technique is applicable to both laboratory and in-situ measurements - for example, as embedded in a cone penetrometer.

\section{APPENDIX}

See Fig. A1-A2.

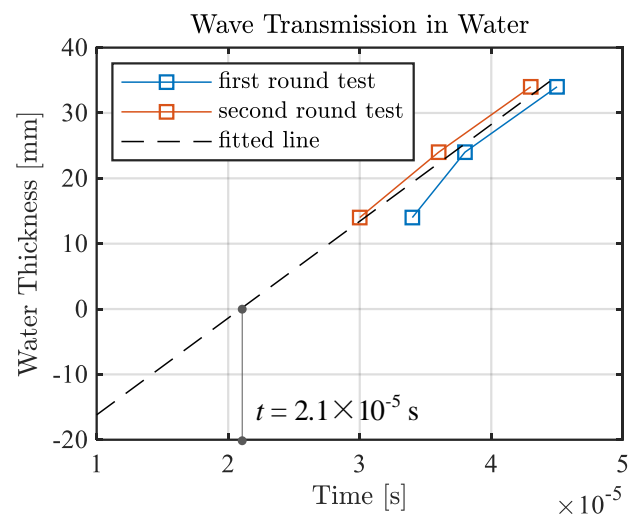

Fig. A1. Using the $40 \mathrm{kHz}$ P-wave transmitter-receiver sensor pair to measure the transmission time in water with respect to the varied water thickness $(14,24$ and $34 \mathrm{~mm})$. The fitted line with two rounds of test results (the slope is the known water wave speed of $1481 \mathrm{~m} / \mathrm{s}$ ) shows a travel time of around $2.1 \times 10^{-5} \mathrm{~s}$ within the sensors themselves.

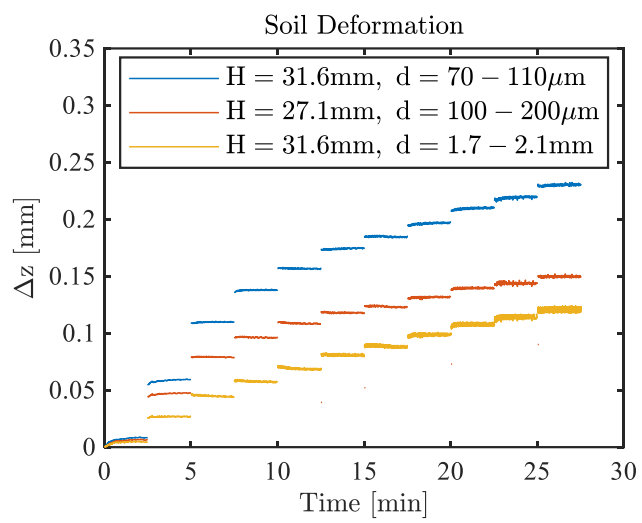

Fig. A2. Representative results of the vertical deformation $\Delta z$ of the soil specimens (glass ballotini) with respect to the time during the loading tests (a fixed timed step of $2.5 \mathrm{~min}$ for each applied load $F=50,100$, $200, \ldots 1000 \mathrm{~N}$ ), showing that the soil density can be taken as constants because of negligible $\Delta z$ : the soil specimens are with the thickness of $H$ $=31.6 \mathrm{~mm}, 27.1 \mathrm{~mm}, 31.6 \mathrm{~mm}$ and the diameters of $d=70-110 \mu \mathrm{m}$, $100-200 \mu \mathrm{m}, 1.7-2.1 \mathrm{~mm}$ respectively.

\section{REFERENCES}

[1] J. C. Santamarina, K. A. Klein, and M. A. Fam, Soils and waves, vol. 316. J. Wiley \& Sons New York, 2001.

[2] S. Dai and J. C. Santamarina, "Stiffness evolution in frozen sands subjected to stress changes," J. Geotech. Geoenvironmental Eng., vol. 143, no. 9, p. 4017042, 2017.

[3] T. Sadek, M. Lings, L. Dihoru, and D. M. Wood, "Wave transmission in Hostun sand: multiaxial experiments," Riv. Ital. Geotec., vol. 41, no. 2, pp. 69-84, 2007.
[4] C. R. I. Clayton, "Stiffness at small strain: research and practice," Géotechnique, vol. 61, no. 1, pp. 5-37, 2011.

[5] G. Alvarado and M. R. COOP, "On the performance of bender elements in triaxial tests," Géotechnique, vol. 62, no. 1, pp. 1-17, 2012.

[6] M. Arroyo, D. Muir Wood, and P. D. Greening, "Source near-field effects and pulse tests in soil samples," Géotechnique, vol. 53, no. 3, pp. 337$345,2003$.

[7] C. Hird and C.-M. Chan, "One-dimensional compression tests on stabilized clays incorporating shear wave velocity measurements," Geotech. Test. J., vol. 31, no. 2, pp. 166-174, 2008.

[8] P. K. Robertson, R. G. Campanella, D. Gillespie, and A. Rice, "Seismic CPT to measure in situ shear wave velocity," J. Geotech. Eng., vol. 112, no. 8, pp. 791-803, 1986.

[9] R. Ghose, "A microelectromechanical system digital 3C array seismic cone penetrometer," Geophysics, vol. 77, no. 3, pp. WA99-WA107, 2012.

[10] H. Dou and P. M. Byrne, "Model studies of boundary effect on dynamic soil response," Can. Geotech. J., vol. 34, no. 3, pp. 460-465, 1997.

[11] M. Lou, W. Wang, T. Zhu, and H. Ma, "Soil lateral boundary effect in shaking fable model test of soil-structure system," Earthq. Eng. Eng. Vib., vol. 4, 2000.

[12] R. Long, T. Vogt, M. Lowe, and P. Cawley, "Measurement of acoustic properties of near-surface soils using an ultrasonic waveguide," Geophysics, vol. 69, no. 2, pp. 460-465, 2004.

[13] Y. Ashida, "Seismic imaging ahead of a tunnel face with three-component geophones," Int. J. Rock Mech. Min. Sci., vol. 38, no. 6, pp. 823-831, 2001.

[14] C.-G. Xie, "Applications of tomography in oil-gas industry-Part 1," in Industrial Tomography, Elsevier, 2015, pp. 591-632.

[15] R. Collins, J. Zhu, R. Mills, and J. Boxall, "Soil Saturation Detection from in Pipe Ultrasound Measurements," 19th World Conference on NonDestructive Testing, 2016.

[16] S.-M. Ham, T.-H. Kwon, I. Chang, and M.-K. Chung, "Ultrasonic P-wave reflection monitoring of soil erosion for erosion function apparatus," Geotech. Test. J., vol. 39, no. 2, pp. 301-314, 2016.

[17] D. Fratta, K. A. Alshibli, W. M. Tanner, and L. Roussel, "Combined TDR and P-wave velocity measurements for the determination of in situ soil density - experimental study," Geotech. Test. J., vol. 28, no. 6, pp. 553563, 2005.

[18] Z. J. Sun, G. D. Young, R. A. McFarlane, and B. M. Chambers, "The effect of soil electrical conductivity on moisture determination using timedomain reflectometry in sandy soil," Can. J. Soil Sci., vol. 80, no. 1, pp. $13-22,2000$.

[19] W. Mao, S. Aoyama, and I. Towhata, "A study on particle breakage behavior during pile penetration process using acoustic emission source location," Geosci. Front., vol. 11, no. 2, pp. 413-427, 2020.

[20] B. J. Zadler, J. H. L. Le Rousseau, J. A. Scales, and M. L. Smith, "Resonant ultrasound spectroscopy: theory and application," Geophys. J. Int., vol. 156, no. 1, pp. 154-169, 2004.

[21] V. T. Rathod, "A Review of Acoustic Impedance Matching Techniques for Piezoelectric Sensors and Transducers," Sensors, vol. 20, no. 14, p. 4051,2020

[22] R. S. Dwyer-Joyce, B. W. Drinkwater, and C. J. Donohoe, "The measurement of lubricant-film thickness using ultrasound," Proc. R. Soc. London. Ser. A Math. Phys. Eng. Sci., vol. 459, no. 2032, pp. 957-976, 2003.

[23] T. Reddyhoff, S. Kasolang, R. S. Dwyer-Joyce, and B. W. Drinkwater, "The phase shift of an ultrasonic pulse at an oil layer and determination of film thickness," Proc. Inst. Mech. Eng. Part J J. Eng. Tribol., vol. 219, no. 6, pp. 387-400, 2005.

[24] S. Kasolang and R. S. Dwyer-Joyce, "Viscosity measurement in thin lubricant films using shear ultrasonic reflection," Proc. Inst. Mech. Eng. Part J J. Eng. Tribol., vol. 222, no. 3, pp. 423-429, 2008.

[25] M. Schirru, R. Mills, R. Dwyer-Joyce, O. Smith, and M. Sutton, "Viscosity measurement in a lubricant film using an ultrasonically resonating matching layer," Tribol. Lett., vol. 60, no. 3, pp. 1-11, 2015.

[26] M. Yu, L. Shen, T. Mutasa, P. Dou, T. Wu, and T. Reddyhoff, "Exact analytical solution to ultrasonic interfacial reflection enabling optimal oil film thickness measurement," Tribol. Int., vol. 151, p. 106522, 2020.

[27] P. Dou, Y. Jia, T. Wu, Z. Peng, M. Yu, and T. Reddyhoff, "High-accuracy incident signal reconstruction for in-situ ultrasonic measurement of oil film thickness," Mech. Syst. Signal Process., vol. 156, 2021, doi: 10.1016/j.ymssp.2021.107669.

[28] N. F. Haines, J. C. Bell, and P. J. McIntyre, "The application of broadband ultrasonic spectroscopy to the study of layered media," J. Acoust. Soc. Am., vol. 64, no. 6, pp. 1645-1651, 1978. 
[29] S. Griffiths, A. Rescaglio, and F. Melo, "Ultrasound propagation in wet and airless non-consolidated granular materials," Ultrasonics, vol. 50, no. 2, pp. 139-144, 2010.

[30] J. D. Goddard, "Nonlinear elasticity and pressure-dependent wave speeds in granular media," Proc. R. Soc. London. Ser. A Math. Phys. Sci., vol. 430 , no. 1878 , pp. $105-131,1990$.

[31] Universal Mechanical Tester (UMT), [Online] Available: https://www.bruker.com/en/products-and-solutions/test-andmeasurement/tribometers-and-mechanical-testers/umt-tribolab.html

[32] Ultrasonic Transducers: Wedges, Cables, Test Blocks. Olympus, Panametrics [Online] Available: https://www.olympus-ims.com

[33] M. Otsubo, C. O'Sullivan, S. Ackerley, and D. Parker, "Selecting an appropriate shear plate configuration to measure elastic wave velocities," Geotech. Test. J., vol. 43, no. 6, 2020.
[34] R. Kuwano and R. J. Jardine, "On the applicability of cross-anisotropic elasticity to granular materials at very small strains," Géotechnique, vol. 52 , no. 10 , pp. $727-749,2002$.

[35] M. Otsubo, R. Kuwano, C. O'Sullivan, and T. Shire, "Using geophysical data to quantify stress transmission in gap-graded granular materials," Géotechnique, pp. 1-18, 2021.

[36] Y. Le Gonidec and D. Gibert, "Multiscale analysis of waves reflected by granular media: Acoustic experiments on glass beads and effective medium theories," J. Geophys. Res. Solid Earth, vol. 112, no. B5, 2007.

[37] R. S. Dwyer-Joyce, B. W. Drinkwater, and A. M. Quinn, "The use of ultrasound in the investigation of rough surface interfaces," J. Trib., vol. 123 , no. 1 , pp. 8-16, 2001. 